\title{
Extracellular Volume Estimation in the Assessment of Myocardial Viability in Ischaemic Cardiomyopathy
}

\author{
Atul Kapur, Goldaa Mahajan, Aprajita Kapur \\ Department of Radiology, Advanced Diagnostics and Institute of Imaging 17/8, Amritsar, Punjab, India \\ Email address: \\ Atulak62kapur@gmail.com (A. Kapur),masatulak@aim.com (G. Mahajan), Aprajita66ak@gmail.com (A. Kapur) \\ To cite this article: \\ Atul Kapur, Goldaa Mahajan, Aprajita Kapur. Extracellular Volume Estimation in the Assessment of Myocardial Viability in Ischaemic \\ Cardiomyopathy. Cardiology and Cardiovascular Research. Vol. 4, No. 3, 2020, pp. 92-98. doi: 10.11648/j.ccr.20200403.13
}

Received: April 30, 2020; Accepted: June 11, 2020; Published: June 28, 2020

\begin{abstract}
OBJECTIVES: To determine the role of extracellular volume estimation (ECV) along with Late gadolinium enhanced (LGE) MRI in assessing viability in patients with chronic ischemic cardiomyopathy. BACKGROUND: Imaging techniques form myocardial viability estimation have shown varying results and outcomes in patients with chronic ischemic cardiomyopathy. In the current form viability estimation is being questioned as a single important prognostic prerevascularisation variable. Hence there is a need to explore new and a robust technique to achieve the above goal. METHODS: 22 consecutive patients diagnosed with chronic ischemic cardiomyopathy which were considered for bypass grafting and had angiographic proven triple vessel disease and or left main stenosis with reduced ejection fraction of $<35 \%$ were enrolled in the study. CMR was done using ECV and LGE protocol. All patients had normal renal functions. Viability (V) scores and Corrected Viability (CV) scores were calculated on LGE and ECV -LGE images. Segments with ECV $>50 \%$ were labeled as nonviable. Six month primary outcome measure was improved ejection fraction following revascularisation. RESULTS: Sensitivity and specificities for detection of nonviable segments on LGE and ECV-LGE were 69\%, 100\%and 96\%, $100 \%$ with AUC's being 0.84 and 0.98 respectively. Patients with CV score of $>8$ showed positive primary outcome of improved ejection fraction of $42.8 \%$ while those with $\mathrm{CV}$ score $<8$ showed a negative primary outcome. Group II patients with viable myocardium with significant fibrous tissue i.e. ECV of $28-49 \%$ showed partially improved function. CONCLUSION: Estimation of ECV-LGE method had $96 \%$ sensitivity in the detection of nonviable segments and also showed a positive primary outcome with improved ejection fraction at six months with viability being a Bayesian variable which depended upon the quantity of fibrous tissue in the viable myocardium.
\end{abstract}

Keywords: Ischemic Cardiomyopathy, Myocardial Viability, Cardiac MRI, Extra Cellular Volume

\section{Introduction}

Ischemic cardiomyopathy is the leading cause of heart failure, repeated hospital admissions and increased mortality with poor quality of life [1]. There is high perioperative mortality $5-35 \% \quad[2,3]$ in patients who underwent vascularisation therefore Viability testing of the myocardium is recommended to determine the presurgical cardiovascular outcome. Dobutamine stress echocardiography, late gadolinium enhancement cardiac MRI (LGE-CMR), 201Thallium, 99TCm sestamibi and 18F-FDG PETMRI are used to address the issue of myocardial viability but have shown varying results $[4,5,6]$. Furthermore the very concept of viability has been questioned after results of various trials. STICH trial [7, 8] based on SPECT studies showed that patients with viable myocardium had lower overall lower death rates but the 5year prognosis status seen after adjustment with prognostic variables like serumcreatinine, diabetes showed no change. PARR-2trial [9, 10] using PET viability assessment showed insignificant reduction in major cardiac events following revascularisation and many patients with viable myocardium did not show appropriate improved ejection fraction. Hence preoperative stratification based on detection of current imaging techniques of myocardial scar detection or perfusion-metabolic mismatch defect alone may not be enough $[11,12]$ and there is a need to improve viability based imaging methods. Cardiac function is a combination of cell metabolism and contractility and therefore addressing the question of viability by mere scar detection or by perfusion metabolic defect be enough to solve the puzzle [13]. A single 
major factor which is connected to pathophysiology of myocardial ischemia is the status of cardiac extracellular matrix or volume-ECV [14]. Changes in the profile of ECV have been implicated in pathogenesis of non ischemic cardiomyopathies [15]. This study was therefore designed to test a) role of a newer imaging technique i.e. myocardial extracellular volume estimation to determine viability. b) To determine if myocardial viability is a dichotomous variable!. To our knowledge no such study has been done so far.

\section{Material and Methods}

The study comprised of 22 consecutive patients of chronic ischemic cardiomyopathy with reduced ejection fraction of less than $35 \%$ with significant angiographic coronary artery stenosis; either triple vessel disease or left main vessel disease. All patients were on medical management with normal renal functions and had NYHA class II, III heart failure with history of angina. CMR was done between January 2017- December 2019 after obtaining informed consent from all the patients. Demographic details of these patients along with their relevant clinical and medical treatment data was recorded (Table 1). All patients underwent reperfusion with coronary artery bypass grafting. The primary outcome measure was improved ejection fraction on a follow up echocardiogram at 6 months.

Table 1. Baseline Characteristics of Patients.

\begin{tabular}{|c|c|c|c|c|c|}
\hline S. No. & Parameter & Group I & Group II & Group II & P value \\
\hline 1 & Number of patients & 4 & 8 & 10 & 0.1 \\
\hline 2 & Age & 55 & 55.5 & 54 & 0.1 \\
\hline \multirow[t]{3}{*}{3} & Sex & & & & $*$ \\
\hline & Males & 4 & 6 & 6 & $*$ \\
\hline & Females & & 2 & 4 & $*$ \\
\hline 4 & Hypertension & 4 & 8 & 3 & * \\
\hline 5 & Family History of CAD & 1 & 5 & 4 & * \\
\hline 6 & $\mathrm{BSA}(\mathrm{m} 2 \mathrm{i})$ & 2.03 & 2.2 & 2.2 & * \\
\hline 7 & Diabetes mellitus & 1 & 3 & 5 & $*$ \\
\hline \multirow[t]{4}{*}{8} & History of pervious MI & 1 & 2 & 4 & $*$ \\
\hline & Dysnoea & & & & $*$ \\
\hline & NYHA I & & 1 & 1 & * \\
\hline & NYHA II & & 3 & 6 & * \\
\hline 9 & NYHA III & 4 & 4 & 3 & * \\
\hline 10 & Mean Ejection fraction $\%$ & 19.5 & 27.5 & 38.6 & 0.05 \\
\hline 11 & Mean end syst. vol index/_VSI $\mathrm{ml} / \mathrm{m} 2$ & 75.2 & 82.1 & 38.5 & 0.01 \\
\hline 12 & Mean end diastoic wall thickness & 5.1 & 6.2 & 7.3 & 0.05 \\
\hline
\end{tabular}

Table 2. Baseline Characteristics on Imaging in Three Groups.

\begin{tabular}{llllll}
\hline GROUPS & CASES & CV SCORE & EF\% & ECV $>\mathbf{2 5 \%}$ & LGE $>\mathbf{5 0 \%}$ \\
\hline I & 4 & 6.1 & 19.5 & 66 & 32 \\
II & 8 & 11 & 27.5 & 45 & 21 \\
III & 10 & 14 & 33.6 & 33 & 15 \\
\hline
\end{tabular}

Table 3. Sensitivity and Specificity analysis of ECV and LGE.

\begin{tabular}{|c|c|c|c|c|c|c|c|}
\hline $\mathbf{n}$ & \multicolumn{3}{|l|}{352} & $\mathbf{n}$ & \multicolumn{3}{|l|}{352} \\
\hline & \multicolumn{3}{|l|}{ SCAR } & & \multicolumn{3}{|l|}{ SCAR } \\
\hline ECV & Present & Absent & Total & LGE & Present & Absent & Total \\
\hline Positive test $>50 \%$ & 102 & 0 & 102 & Positive test $>0$ & 68 & 0 & 68 \\
\hline Negative test $\leq 50 \%$ & 4 & 246 & 250 & Negative test $\leq 0$ & 34 & 250 & 284 \\
\hline Total & 106 & 246 & 352 & Total & 102 & 250 & 352 \\
\hline Sample Prevalence & 0.300 & $95 \% \mathrm{CI}$ & & Sample Prevalence & & $\begin{array}{l}0.300 \\
95 \% \mathrm{CI}\end{array}$ & \\
\hline Sensitivity - TP proportion & 0.962 & 0.906 to & & Sensitivity - TP proportion & 0.689 & 0.591 to & \\
\hline Specificity - TN proportion & 1.000 & 0.985 to & & Specificity - TN proportion & 1.000 & 0.985 to & \\
\hline FP proportion & 0.000 & 0.000 to & & FP proportion & 0.000 & 0.000 to & \\
\hline FN proportion & 0.038 & 0.010 to & & FN proportion & 0.311 & 0.225 to & \\
\hline
\end{tabular}

\subsection{CMR Protocol}

Informed consent was obtained from all the patients being undergoing the examination. Patients were positioned in supine on a 1.5 Tesla Cardiac MRI scanner ( Siemens Amira, Shenzen, China) using a 16 channel phased array surface coil with EKG gating. Cine images of the heart were taken from base to the apex in short axis, 4 chamber views. Pre contrast
T1 maps of the heart were obtained in the mid, basal and apical short axis views using Modified Look-Locker inversion recovery sequence (MOLLI). A perfusion study was then done in short axis views at the three sites as described using Intravenous $0.15 \mathrm{mmol} / \mathrm{Kg}$ gadolinium contrast (Multihance Bracco, Singen Germany) bolus injection. Post contrast T1 maps were obtained in the similar positions as the plain study at 10 minutes interval. This was 
followed by Phase contrast inversion recovery sequence for LGE if any of the left ventricle from the base to the apex of left ventricle.

\subsection{Image Analysis}

Was done by experienced cardiac radiologists to record for wall motion abnormalities of the left ventricle, areas of LGE enhancement, thickness of the myocardium and percentage of scar tissue. The pre and post contrast T1 maps were processed for ECV maps using (CMR Segment software, University of Lund, Sweden). The left ventricle myocardium was divided into 16 segments based on AHA model. LGE images were assessed for enhancing scars. Focal scars with more than $50 \%$ thickness of myocardium were labeled as noviable with score o, normal myocardial segments or those with less than $50 \%$ scar thickness were labeled as viable and given score 1 and a $\mathrm{V}$ (viability) score computed for all 16 segments. ECV maps were assessed for quantitative volume in percentages. ECV $<25$ was normal- score 0 , segments with increased ECV up till $49 \%$-score 0.5 and segments with $\mathrm{ECV}>/=50 \%$ were given score of -1 . Corrected segmental scores were added to formulate a corrected Viability (CV) score maximum score being 16. Based on CV scores patients were classified into thress groups: Group I with CV score $<8$ and had insignificant viable myocardium, group II with $\mathrm{CV}$ score of 8-12 and group III with CV scores 12-16 group III.

\subsection{Statistical Analysis}

Was done using Analyse -IT software (Leeds UK) and ECV map

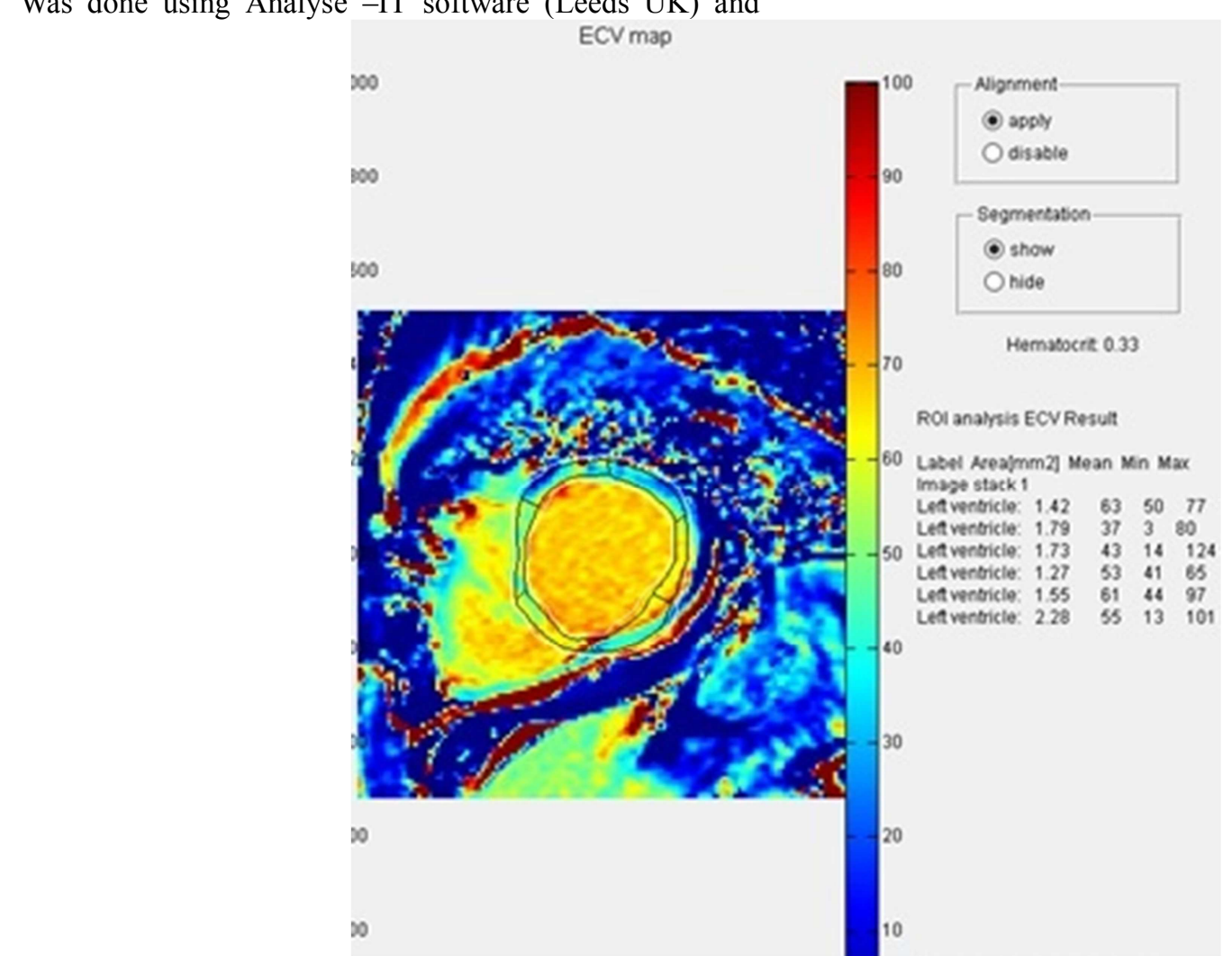

continous variables were compared using student t test, sensitivity, specificity and true and false positives were calculated along with likelihood ratios for both the techniques. AUC was estimated for ECV and LGE techniques for estimation of predicting myocardial viability. Post Hoc analysis was done to determine power of the tests. Results of follow up Left ventricle function determined by routine transthoracic echogardiography at 6 months were compared with baseline viability scores.

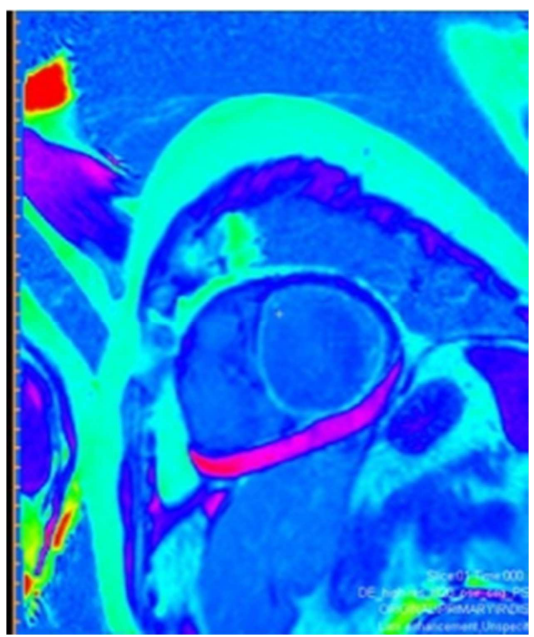

Figure 1. Late gadolinium enhancement image in short axis at mid left ventricle showing transmural and subendocardial enhancing scar with viability score of 10 .

Figure 2. ECV map showing increased ECV $>50 \%$ in three segments in mid left ventricle with no change in viability score with CV score 10. 


\section{Results}

22 consecutive patients of established coronary artery disease with ischemic cardiomyopathy with reduced ejection fraction underwent CMR using the technique described. The mean age of the patients was 55 years (48-61 years). 16 were males and 6 were females. The mean ejection fraction was $29.2 \%(20.3-36.1) 98.3 \%$ CI (Table 1). Out of the 352 left ventricle segments examinedbyLGE284segments showed no LGE or scar tissue less than 50\%thickness of myocardiumviable while 68 segments had LGE which was more than $50 \%$. Mean Vscorewas 12 by LGE (Figures1, 2). ECV of more than $50 \%$ (non viable segments) was seen in 102 segments, while ECVof28-49\%was seen in 42 segments, 208 segments had normal ECV. The baseline characteristics of three groups are shown in (Table 2) which showed a mean CV scores was of 6.1, 11 and 14.0 respectively (pvalue 0.05 ) (Figures 3, 4). Four segments which had microvascular obstruction MVO seen on contrast images and showed false low $\mathrm{T} 1$ values (Figures 5,6 ). The sensitivity and specificity for estimation of nonviable myocardium based on LGE was $69 \%, 100 \%$ with a false negative of $31 \%$ compared to $96 \%$ and $100 \%$ using both LGE and by ECV (Table 3). The AUC's for LGE and ECV was 0.84 and 0.98 respectively (p value 0.003 ) (Figure 7). Post hoc analysis of the above tests showed a power of 0.78 . MeanT1 map value was 1070 (1031-1117; 95\%CI) msec in myocardial segments which did not show any LGE and had a ECV of less than $50 \%$. Those segments with LGE more than $50 \%$ had a mean T1 of 1161 (1105-1211; 95\% CI) msec the differences being statistically significant ( $p$ value 0.004). Six months follow up echocardiography showed a positive primary outcome in group III, II patients with $\mathrm{CV}>8 \%$ and had improved mean resting ejection fraction of $42.8 \%, 36.1 \%$ respectively while no significant change in ejection fraction was seen in patients with CV scores of $<8$ in group. (Figure 8 ).

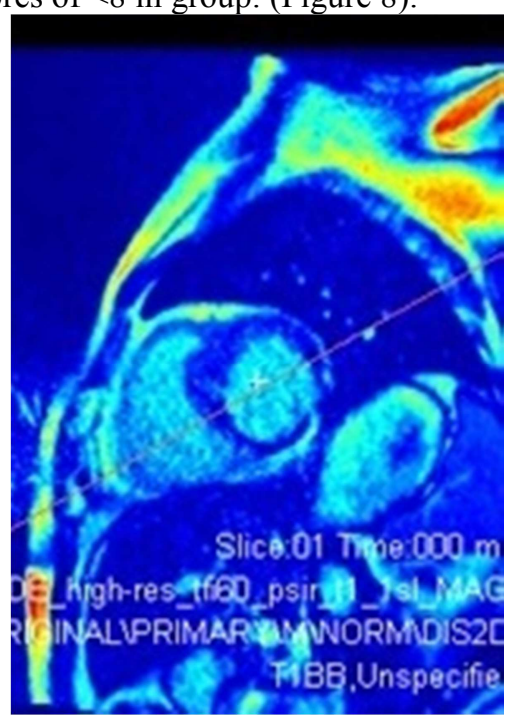

Figure 3. Short axis view of late gadolinium enhancement image showing no $L G E$ with thinning of lateral wall in mid left ventricle level with a viability score of 16.

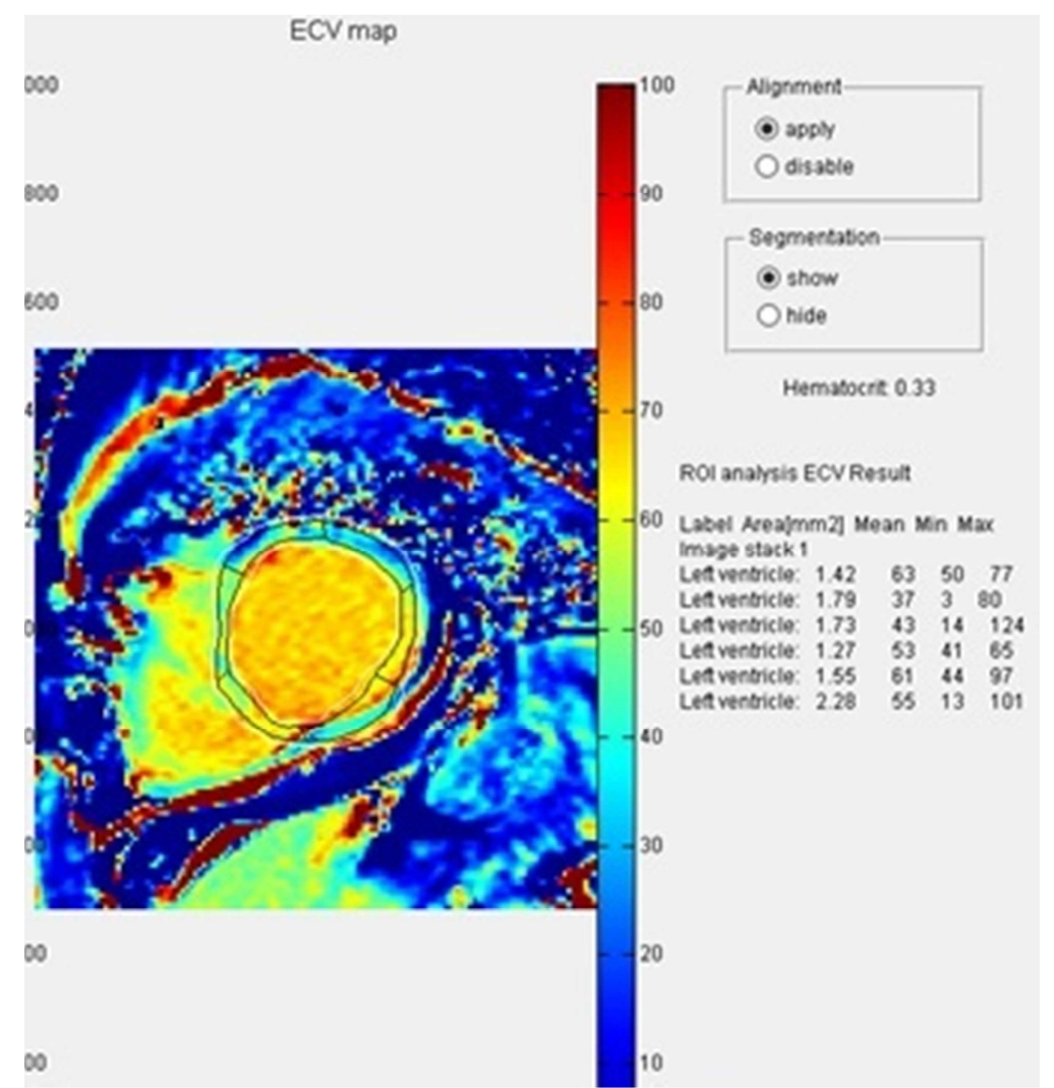

Figure 4. ECV map of same patient showing segments with increased ECV $>50 \%$ with corrected viability score of 8 . 


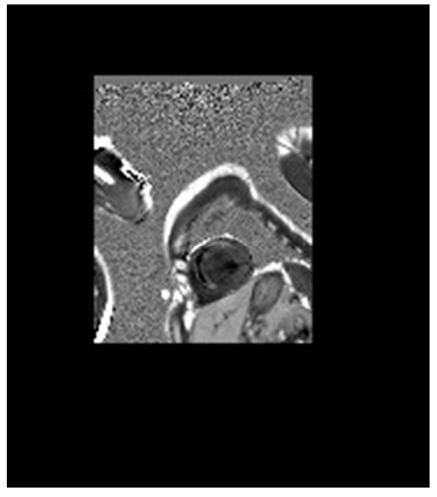

Figure 5. LGE image showing transmural infarct in mid interventricular septum with internal hypointensity due to microvascular obstruction.

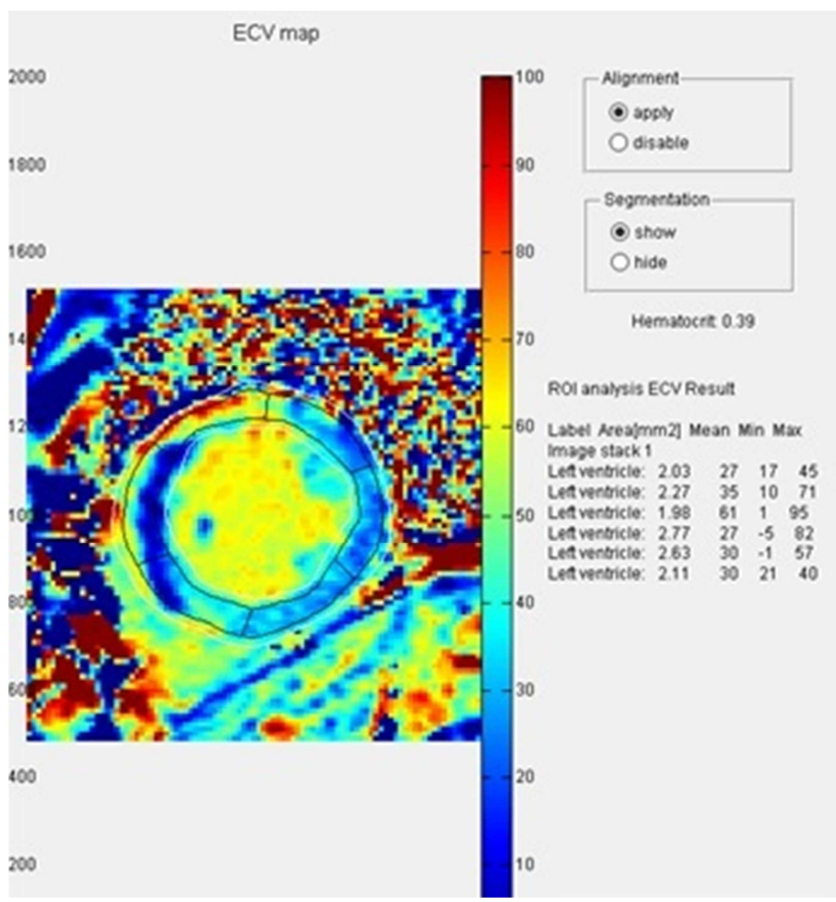

Figure 6. ECV map of the same patient showing reduced ECV in MVO.
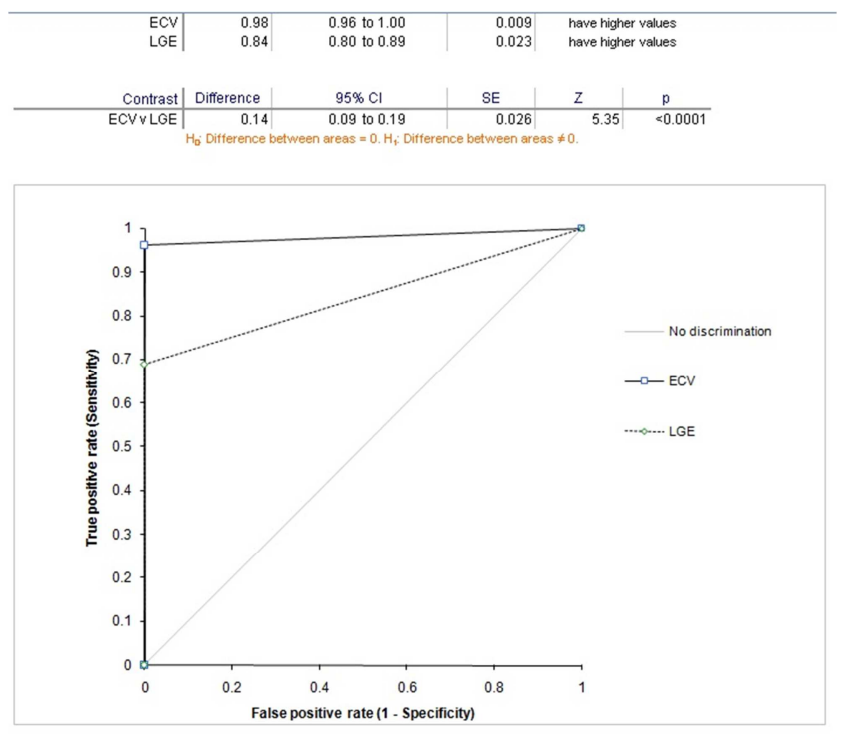

Figure 7. Area under curve of ECV and LGE CMR for assessing viability.

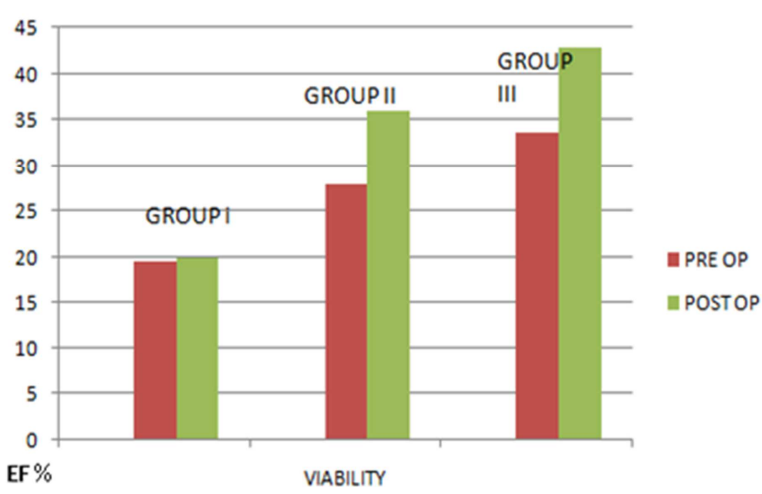

Figure 8. Bar chart showing comparative changes in ejection fraction post revascularisation in three groups.

\section{Discussion}

In spite of the limitations to predict functional improvement and long term outcome, viability assessment is important in the evaluation of patients with ischemic cardiomyopathy before surgical revascularization [16]. LGECMR and 18F-FDG PET have been so far the preferred tools due to increased sensitivity compared with dobutamine echocardiography which only assesses contractile reserve [17]. Our study showed improved sensitivity and specificity of $96 \%$ and $100 \%$ of LGE- CMR when combined with ECV for detection of non viable myocardial segments when compared with LGE-CMR alone which had a sensitivity of $69 \%$ with high a false positive rate $(31 \%)$. Existing technique of LGE-CMR alone fails to detect diffuses everely fibrosed dysfunctional hibernating myocardium due to lack of gadolinium uptake is diagnosed as viable. The se are usually segments with cardiac remodeling with or without underlying subendocardial infarcts with variable fibrous tissue and show variable functional recovery as seen in group II patients in the current study. Similar results were seen in STICH triaI and PARR-2 trial with SPECT and PET CT where segments labelled viable did not show complete functional recovery $[7,8]$. By clinical definition "a viable myocardium is one without significant fibrosis and where its function is expected to improve following revascularisation [18]. This study tested the the traditional concept of viability as a dichotomous variable i.e Either viable or non viable by dividing patients into three groups i.e those with non viable myocardium (group I), viable myocardium (group III) and a intermediate group II with viable with incomplete recovery and showed statistically significant differences between them. Our study shows that Viability is a continuum of these three states rather than a dichotomous variable and this is based on the amount interstitial fibrosis or extracellular matrix in the myocardium which so far could not be detected by available techniques in imaging including 18-F FDG PET. Many segments of myocardium with significant scarring are deemed as viable due to presence of low perfusion and metabolic active state and do not show functional recovery post reperfusion. These segments are falsely deemed viable 
based on LGE-CMR alone and PET as seen in our study which showed a false positive of $31 \%$ viable segments which had absent enhancement. This could be the reason of poor long term prognosis of current viability techniques post revascularization [7]. Variables like coexisting diabetes, serum creatinine and obesity in a multivariate regression models factors also effect myocardial interstitium [19]. These play important role in cardiac adverse remodeling and pathophysiology of heart failure. It is likely that recovery of cardiac function following revascularization in ischemic cardiomyopathy also involves ECV pathways and those patients which recover better have reduced matrix degradation fibroblasts, reduced metalloproteins and less fibrous tissue [21] while. segments with increased ECV show poor microvascular function and neoangiogenesis which in turn impairs the functional recovery and inhibits positive remodeling as seen in. Group I patients of our study. Patients in group II showing increased ECV but are viable and show partial recovery of function due to higher fibrosis compared to the patients with Group III. Viability therefore appears to be bayesian in nature rather than a dichotomous variable [22]. CV Scores obtained using both LGE and ECV was a statistically better parameter for viability assessment and influenced the primary outcome measure of improved ejection fraction at 6 months post revascularization.

The potential limitation of study was that we determined only improved ejection fraction as the primary outcome measure and did not determine the clinical and long term outcome.

\section{Conclusion}

To conclude use of combined ECV-LGE CMR improves the sensitivity of detection of nonviable segments to $96 \%$ and had a better correlation with short term primary out come measure of improved ejection fraction in patients with chronic ischemic cardiomyopathy post revascularization. It has the potential to be single short and long term prognostic variable in the evaluation of such patients. Viability is not a dichotomous variable but a continuum of viable, moderately viable and an on viable states which can be determined using this technique.

\section{References}

[1] Baker D, Jones R, Hodges J, et al. Management of Heart Failure. III. The role of revascularization in the treatment of patients with moderate or severe left ventricular systolic dysfunction. JAMA. 1994; 272: 1158-1134.

[2] Coronary artery surgery study (CASS): a randomized trial of coronary artery bypass surgery. Survival data. Circulation1983; 68: 939-50. 10.1161/01.CIR.68.5.939.

[3] Travin MI, Bergmann SR. Assessment of myocardial viability. Semin Nucl Med. 2005; 2: 2-19.

[4] Bruder O. Wagner A. Jensen C. J., et al. Myocardial scar visualized by cardiovascular magnetic resonance imaging predicts major adverse events in patients with hypertrophic cardiomyopathy. J Am Coll Cardiol. 2010. 56: 875-887.

[5] Kidambi A, Motwani M, Uddin A, Ripley DP, etal. Myocardial Extracellular Volume Estimation by CMR Predicts Functional Recovery Following Acute MI. JACC 2016.10: 989-999.

[6] Ichiro M, Junichi T, Kenichi N, Norihisa T, Kinichi H. Myocardial viability assessment using nuclear imaging. Ann Nucl Med. 2003; 17: 169-179.

[7] Velazquez EJ, Lee KL, O'Connor CM, et al. The rationale and design of the Surgical Treatment for Ischemic Heart Failure (STICH) trial. J Thorac Cardiovasc Surg 2007; 134: 1540-7.

[8] Velazquez EJ, Lee KL, Deja MA, et al. Coronary-artery bypass surgery in patients with left ventricular dysfunction. N Engl J Med2011; 364: 1607-16.

[9] Abraham A, Nichol G, Williams KA, Guo A, de Kemp RA, Garrard L, et al; PARR 2 Investigators. 18F-FDG PET imaging of myocardial viability in an experienced center with access to $18 \mathrm{~F}-\mathrm{FDG}$ and integration with clinical management teams: the Ottawa-FIVE substudy of the PARR 2 trial. J Nucl Med. 2010; 51 (4): 567-74.

[10] Beanlands R. S. B., Nichol G., Huszti E., et al. PARR-2 Investigators. F-18-fluorodeoxyglucose positron emission tomography imaging-assisted management of patients with severe left ventricular dysfunction and suspected coronary disease: a randomized, controlled trial (PARR-2) J Am Coll Cardiol. 2007; 50: 2002-2012.

[11] Kim RJ, Shah DJ. Fundamental concepts in myocardial viability assessment revisited: when knowing how much is "alive" is not enough. Heart 2004; 90: 137-140.

[12] Ramos M, De Pasquale E, Coplan NL. Assessment of myocardial viability: review of the clinical significance. Rev Cardiovasc Med. 2008; 9: 225-231.

[13] Beller GA, Gimple LW. Myocardial viability. Assessment by cardiac scintigraphy. Cardiol Clin. 1994 May; 12 (2): 317-32.

[14] Wilter SK, Nunes TP, Nacif MS, Mesquita T. Practical Implications of Myocardial Viability Studies. Arq Bras Cardiol. 2018; 110 (3): 278-288.

[15] Miller CA, Naish JH, Bishop P, Coutts G, Clark D, Zhao S, Ray SG, Yonan N, Williams SG, Flett AS, et al.. Comprehensive validation of cardiovascular magnetic resonance techniques for the assessment of myocardial extracellular volume. Circ Cardiovasc Imaging. 2013; 6: 373383.

[16] Puntmann VO, Carr-White G, Jabbour A, Yu CY, Gebker R, Kelle S, Hinojar R, Doltra A, Varma N, Child N, et al. T1Mapping and Outcome in Nonischemic Cardiomyopathy: AllCause Mortality and Heart Failure. JACC Cardiovasc Imaging. 2016 Jan; 9 (1): 40-50.

[17] Pellikka PA, Nagueh SF,. Elhendy AA, Kuehl CA, Sawada SG. "American Society of Echocardiography recommendations for performance, interpretation, and application of stress echocardiography," Journal of the American Society of Echocardiography 2007; 20: 9, 10211041.

[18] Gropler RJ, Bergman SR. Myocardial Viability "What Is the Definition”. J Nucl Med. 1991; 32: 10-12. 
[19] Bax JJ, Poldermans D. Clinical value of assessment of perfusion and function for the evaluation of myocardial viability in patients with ischemic left ventricular dysfunction. In: Germano G, Berman DS, eds. In: Clinical Gated Cardiac SPECT 2nd ed. Massachusetts: Blackwell Publishing Ltd; 2006: 260.

[20] Treasure CB, Klein JL, Vita JA, et al. Hypertension and left ventricular hypertrophy are associated with impaired endothelium-mediated relaxation in human coronary resistance vessels. Circulation. 1993; 87: 86-93.

[21] Frangogiannis NG. The Extracellular Matrix in Ischemic and Nonischemic Heart Failure. Circulation Research 2019; 117146.

[22] Redfors B, Stone GW. Myocardial viability and CABG surgery: a Bayesian appraisal of STICH. Nat Rev Cardiol. 2019 Dec; 16 (12): 702-703. 